\title{
CONTINUITY OF ADDITIVE $\kappa$-METRIC FUNCTIONS AND METRIZATION OF $\kappa$-METRIC SPACES
}

\author{
TAKESI ISIWATA
}

(Communicated by Dennis K. Burke)

\begin{abstract}
For an additive $\kappa$-metric space $X$ with an $s(x)$-continuous $\kappa$ metric $d(x, C)$, we prove that $X$ is metrizable, and that if $d(x, C)$ is locally regular, then $z(x, y)$ is bicontinuous, and $\rho(x, y)=z(x, y)+z(y, x)$ is a metric on $X$ which agrees with the topology of $X$.
\end{abstract}

Introduction. Dranišnikov [2] defined the additivity of a $\kappa$-metric space, which is introduced by Šepin [4,5] as a generalization of metric spaces and locally compact groups, and stated that a compact additive $\kappa$-metric space is metrizable. In the previous paper [3], we proved that an additive $\kappa$-metric space $X$ is metrizable if $X$ is either pseudocompact or stratifiable. In this paper, investigating the relationship among various kinds of continuities of a $\kappa$-metric $d(x, C)$, we prove the following:

MAIN THEOREM. Let $(X, d)$ be an additive $\kappa$-metric space with an $s(x)$-continuous $\kappa$-metric $d(x, C)$. Then we have:

(1) $X$ is metrizable.

(2) If $d(x, C)$ is locally regular, then $z(x, y)$ is bicontinuous and $\rho(x, y)=z(x, y)+$ $z(y, x)$ is a metric on $X$ which agrees with the topology of $X$.

Throughout this paper, we mean by $(X, d)$ an additive $\kappa$-metric space.

\section{Definition and preliminaries.}

1.1. DEFinition. Let $X$ be a topological space and let $\operatorname{Rc}(X)$ be the collection of regular closed subsets of $X .(X, d)$ is an additive $\kappa$-metric space $[2,4,5]$ if $X$ is Tychonoff and $d$ is a mapping of $X \times \operatorname{Rc}(X)$ to $[0,1]$ satisfying the following:

(1) $d(x, C)=0 \Leftrightarrow x \in C$.

(2) $C \subset D \Rightarrow d(x, C) \geq d(x, D)$.

(3) $d(x, C)$ is continuous with respect to $x$ for each $C$ (abbreviated by $x$-continuous).

(4) $d\left(x, \operatorname{cl}\left(\bigcup\left\{C_{a} ; a \in A\right\}\right)\right)=\inf \left\{d\left(x, C_{a}\right) ; a \in A\right\} . \quad d(x, C)$ is called an additive $\kappa$-metric function (abbreviated by additive $\kappa$-metric) on $X$. For a closed set $F$ of $(X, d)$, we put $\operatorname{Rc}(F, X)=\{C \in \operatorname{Rc}(X) ; F \subset C\}, \operatorname{Rci}(X, F)=\{C \in$ $\operatorname{Rc}(X) ; F \subset$ int $C\}, \operatorname{Rci}(x)=\operatorname{Rci}(X,\{x\}), \operatorname{Ro}(X)=\{U ; U$ is regular open $\}$, $z(x, F)=\sup \{d(x, C) ; C \in \operatorname{Rci}(X, F)\}$, and $\mathbf{N}=$ the set of positive integers. We introduce in $\operatorname{Rc}(X)$ the Vietoris topology in the following sense: the basic open sets in $\operatorname{Rc}(X)$ are the sets of the form $\left\langle U_{1}, U_{2}, \ldots, U_{n}\right\rangle$ where $U_{i} \in \operatorname{Ro}(X), 1 \leq i \leq n$, and $\left\langle U_{1}, U_{2}, \ldots, U_{n}\right\rangle=\left\{F \in \operatorname{Rc}(X) ; F \subset \cup U_{i}\right.$ and $F \cap U_{i} \neq \varnothing$ for each $\left.i\right\}$. It

Received by the editors September 8, 1986 and, in revised form, December 17, 1987.

1980 Mathematics Subject Classification (1985 Revision). Primary 54C05, 54E15, 54E35, 54E99.

Key words and phrases. Metrization, additive $\kappa$-metric, Vietoris topology, continuous maps. 
is easily verified that $\operatorname{Rc}(X)$ is Hausdorff. We say that $d(x, C)$ is $C$-continuous (bicontinuous) if $d(x, C)$ is continuous with respect to $C$ (jointly continuous with respect to $x$ and $C) . d(x, C)$ is regular [4] if it satisfies the following:

(R) $d(x, C) \leq d(x, D)+\sup \{d(s, C) ; s \in D\}$ for $x, C$ and $D$. We say that $d(x, C)$ is locally regular if for $x, y, t$ and $\varepsilon>0$, there are $C_{0} \in \operatorname{Rci}(y)$ and $D_{0} \in \operatorname{Rci}(t)$ such that

(LR) $d(x, C) \leq d(x, D)+\sup \{d(s, C) ; s \in D\}+\varepsilon$ for $C \in \operatorname{Rci}(y)$ with $C \subset C_{0}$ and $D \in \operatorname{Rci}(t)$ with $D \subset D_{0}$.

It is evident that if $d(x, C)$ is regular, it is locally regular. We say that $d(x, C)$ is $s(x)(s(y)$ and $s(x, y)$, resp.) -continuous if for $\varepsilon>0, x$ and $y$ there are $U \in \operatorname{Rci}(x)$ and $C \in \operatorname{Rci}(y)$ satisfying the following condition $((s(x)),((s(y))$ and $(s(x, y))$, resp.).

$(s(x))\left|d(x, D)-d\left(x^{\prime}, D\right)\right|<\varepsilon$ for $x^{\prime} \in U$ and $D \in \operatorname{Rci}(y)$ with $D \subset C$.

$(s(y))|d(x, D)-d(x, E)|<\varepsilon$ for $D \in \operatorname{Rci}(y)$ with $D \subset C$ and $E \in \operatorname{Rc}(X)$ with $E \subset C$.

$(s(x, y))\left|d(x, D)-d\left(x^{\prime}, E\right)\right|<\varepsilon$ for $x^{\prime} \in U, D \in \operatorname{Rci}(y)$ with $D \subset C$ and $E \in \operatorname{Rc}(X)$ with $E \subset C$.

1.2 LEMMA. For $(X, d)$ we have

(1) $z(x, F)=0 \Leftrightarrow x \in F$, and $z(x, F)=\inf \{z(x, y) ; y \in F\}[3]$.

(2) $z(x, C) \leq d(x, C)$ for $C \in \operatorname{Rc}(X)$.

(3) $\{U(x, n) ; n \in \mathbf{N}\}$ is an open base at $x$ where $U(x, n)=\operatorname{int} S(x, n)$ and $S(x, n)=\{t ; z(x, t)<1 / n\}$.

(4) For $\varepsilon>0, x$ and $y$, there are $C \in \operatorname{Rci}(x)$ and $D \in \operatorname{Rci}(y)$ such that $z(x, y)-$ $\varepsilon<z\left(x^{\prime}, y^{\prime}\right)$ for $x^{\prime} \in C$ and $y^{\prime} \in D$.

PROOF. (4) Let $x \neq y$. By the definition of $z(x, y)$, there is $E \in \operatorname{Rci}(y), x \notin E$ with $z(x, y)-\varepsilon / 2<d(x, E)$. Since $d(x, E)$ is $x$-continuous, there is $C \in \operatorname{Rci}(x)$ such that $\left|d(x, E)-d\left(x^{\prime}, E\right)\right|<\varepsilon / 2$ for $x^{\prime} \in C$. If $y^{\prime} \in$ int $E, d\left(x^{\prime}, E\right) \leq z\left(x^{\prime}, y^{\prime}\right)$ and hence $z(x, y)-\varepsilon<z\left(x^{\prime}, y^{\prime}\right)$ for $x^{\prime} \in C$ and $y^{\prime} \in D \in \operatorname{Rci}(y)$ with $D \subset$ int $E$.

2. Example. In this section, we show that the Sorgenfrey line $\mathbf{R}$ is a nonmetrizable additive $\kappa$-metric space such that $d(x, C)$ is regular and bicontinuous, but not $s(x)$-continuous. For $x \in \mathbf{R}$ and $C \in \mathbf{R c}(\mathbf{R})$, put $C\left(x^{+}\right)=C \cap[x, \infty)$ and $C^{+}(x)=\inf \left\{t \in C\left(x^{+}\right)\right\}(=a)$ if $C\left(x^{+}\right) \neq \varnothing$. We define $d(x, C)=\min \{a-x, 1\}$ if $C\left(x^{+}\right) \neq \varnothing$, and $d(x, C)=1$ otherwise. In [6] it is proved that $(\mathbf{R}, d)$ is an additive $\kappa$-metric space, and $d(x, C)$ is regular.

(1) $d(x, C)$ is bicontinuous. Let $x \in \mathbf{R}$ and $C \in \mathrm{Rc}(\mathbf{R})$.

Case 1. $C\left(x^{+}\right)=\varnothing$. Then $d(x, C)=1$. Put $U=(-\infty, x)$ and $V=[x, x+1)$. Then $d\left(x^{\prime}, D\right)=1$ for $x^{\prime} \in V$ and $D \in\langle U\rangle$, and hence $d(x, C)=d\left(x^{\prime}, D\right)$.

Case 2. $C\left(x^{+}\right) \ni x$. Then $d(x, C)=0$. Since $\operatorname{cl}($ int $C) \ni x$ for a given $\varepsilon>0$, $A=[x, x+\varepsilon) \cap C \neq \varnothing$. Take $\delta>0$ and $y \in A$ such that $y-x>\delta>0$ and $[y-\delta, y+\delta] \subset(x, x+\varepsilon)$. Put $U_{1}=(-\infty, y-\delta), U_{2}=[y-\delta, y+\delta)$, $U_{3}=[y+\delta, \infty)$, and $U=[x, y-\delta)$. For $D \in\left\langle U_{1}, U_{2}, U_{3}\right\rangle$ and $t \in U$. We have $d(t, D) \leq d\left(t, D \cap U_{2}\right)<\varepsilon$, and hence $|d(t, D)-d(x, C)|<\varepsilon$.

Case 3. $C\left(x^{+}\right) \neq \varnothing$ and $C\left(x^{+}\right) \not \supset x$. Let $0<2 \varepsilon<\min \{1, a-x\}$. Put $U_{1}=(-\infty, x), U_{2}=[a-\varepsilon, a+\varepsilon), U_{3}=[a+\varepsilon, \infty)$ and $U=[x, x+\varepsilon)$. For $t \in U$ and $D \in\left\langle U_{1}, U_{2}, U_{3}\right\rangle, D \cap U_{2} \neq \varnothing$, and $a-x-\varepsilon<d(t, D) \leq d\left(t, U_{2} \cap D\right) \leq$ $\min \{1,(a+\varepsilon)-x\}$, and hence $|d(t, D)-d(x, C)|<\varepsilon$. 
(2) $d(x, C)$ is not $s(x)$-continuous. Let $x=y$. For any open set $U \ni x$, take $D \in \operatorname{Rci}(y)$ with $D \subset C$ and $x^{\prime} \in U-D$. Then $d\left(x^{\prime}, D\right)=1$ and $d(x, D)=0$, and hence the condition $(s(x))$ in 1.1 does not hold.

\section{Local regularity and $C$-continuity of $d(x, C)$.}

3.1. THEOREM. For $(X, d)$, the following are equivalent.

(1) $d(x, C)$ is $C$-continuous.

(2) $z(x, C)=d(x, C)$ for $C \in \operatorname{Rc}(X)$.

(3) $z(x, y)=\sup \{d(x, C) ; y \in C \in \operatorname{Rc}(X)\}$.

(4) $d(x, C)$ is $s(y)$-continuous.

(5) $z(x, y)$ is $y$-continuous.

ProOF. (1) $\Rightarrow(2)$. For $\varepsilon>0$ and $C \in \operatorname{Rc}(X)$, there is an open set $\left\langle U_{1}, U_{2}, \ldots, U_{n}\right\rangle$ $\ni C$ such that $|d(x, C)-d(x, D)|<\varepsilon$ for $D \in\left\langle U_{1}, U_{2}, \ldots, U_{n}\right\rangle$. Since $X$ is a $\kappa$-metric space, we can take $D \in \operatorname{Rci}(X, C) \cap\left\langle U_{1}, U_{2}, \ldots, U_{n}\right\rangle$. But $d(x, C)<d(x, D)+\varepsilon$ implies $d(x, C)<z(x, C)+\varepsilon$ by the definition of $z(x, C)$, so $d(x, C) \leq z(x, C)$. By 1.2(2), we have $d(x, C)=z(x, C)$.

$(2) \Rightarrow(3) . \operatorname{Sup}\{d(x, C) ; y \in C \in \operatorname{Rc}(X)\}=\sup \{\sup \{d(x, D) ; D \in \operatorname{Rci}(X, C)\} ;$ $C \in \operatorname{Rc}(X)\}=\sup \{d(x, C) ; C \in \operatorname{Rci}(y)\}=z(x, y)$.

$(3) \Rightarrow(4)$. Let $\left\{C_{n} \in \operatorname{Rci}(y) ; n \in \mathbf{N}\right\}$ be a base at $y$ with $C_{n+1} \subset C_{n}$, and $a=z(x, y)$. Then $a=\lim d\left(x, C_{n}\right)$, and hence we can assume that $a-\varepsilon / 2<$ $d\left(x, C_{n}\right) \leq a$ for $n \in \mathbf{N}$. Suppose that (4) does not hold. Then for each $n$, $\left|d\left(x, D_{n}\right)-d\left(x, E_{n}\right)\right| \geq \varepsilon$ for some $D_{n} \in \operatorname{Rci}(y)$ with $D_{n} \subset C_{n}$ and some $E_{n} \subset C_{n}$. Since $d\left(x, C_{n}\right) \leq d\left(x, D_{n}\right) \leq a$ and $d\left(x, C_{n}\right) \leq d\left(x, E_{n}\right)$, we have $d\left(x, E_{n}\right)>a+\varepsilon / 2$. On the other hand, $y \in \operatorname{cl}\left(\bigcup E_{n}\right)(=A)$ and $d(x, A)=\inf \left\{d\left(x, E_{n}\right) ; n \in \mathbf{N}\right\} \geq$ $a+\varepsilon / 2$, but $a=z(x, y) \geq d(x, A)$ by (3), a contradiction.

$(4) \Rightarrow(5)$. Applying $E \in \operatorname{Rci}\left(y^{\prime}\right)$ to $E$ in $(s(y))$ of 1.1 where $y^{\prime} \in$ int $C, \mid z(x, y)-$ $z\left(x, y^{\prime}\right) \mid \leq \varepsilon$, and hence $z(x, y)$ is $y$-continuous.

$(5) \Rightarrow(2)$. Let $C \in \operatorname{Rc}(X)$ and $x \in X$. By 1.2(1,2), $d(x, C) \geq z(x, C)=$ $\inf \{z(x, t) ; t \in C\}$. Since $z(x, y)$ is $y$-continuous, we have $z(x, C)=\inf \{z(x, t)$; $t \in$ int $C\}$. But $C \in \operatorname{Rci}(t)$ implies $z(x, t) \geq d(x, C)$, and hence $z(x, C) \leq d(x, C)$, so $d(x, C)=z(x, C)$.

$(5) \Rightarrow(1)$. Let $x \in X, \varepsilon>0$ and $a=d(x, C)$. By (5) $\Rightarrow(2)$, and (5), we have $d(x, C)=z(x, C)=\inf \{z(x, y) ; y \in C\}=\inf \{z(x, y) ; y \in \operatorname{int} C\}$, and hence there is $y \in \operatorname{Int} C$ with $a \leq z(x, y)<a+\varepsilon$. Let us put $U=\{t ; z(x, t)>a-\varepsilon\}$ and $W=\{t ; a-\varepsilon<z(x, t)<a+\varepsilon\}$. Then $U \supset W \cup C$. Take $S \in \operatorname{Rci}(y)$ with $S \subset U_{1}=W \cap$ int $C$ and put $U_{2}=W \cap(X-S)$. Then $C \in\left\langle U_{1}, U_{2}\right\rangle$. Let $D \in\left\langle U_{1}, U_{2}\right\rangle$. Put $D_{i}=D \cap \operatorname{ll} U_{i}, i=1,2$. Then $D=D_{1} \cup D_{2}$, and $D, D_{i} \in \operatorname{Rc}(X)$, $i=1$, 2. For $t \in U_{1} \cap D, a-\varepsilon<z(x, t)<a+\varepsilon$, and for $t \in U_{2} \cap D, a-\varepsilon<z(x, t)$. Since $z(x, D)=d(x, D)=\min \left\{d\left(x, D_{i}\right) ; i=1,2\right\}=\min \left\{\inf \left\{z(z, t) ; t \in U_{i} \cap D\right\}\right.$; $i=1,2\}$, we have $a-\varepsilon<d(x, D)<a+\varepsilon$, i.e., $|d(x, D)-d(x, C)|<\varepsilon$, and hence $d(x, C)$ is $C$-continuous.

3.2. THEOREM. For $(X, d)$, we have $(1) \Leftrightarrow(2) \Rightarrow(3)$.

(1) $d(x, C)$ is locally regular.

(2) $z(x, y) \leq z(x, t)+z(t, y)$ for $x, y$ and $t$.

(3) $z(x, y)$ is $y$-continuous. 
ProOF. (1) $\Rightarrow(2)$. Suppose that (LR) in 1.1 holds. Let $x, y, t \in X, D_{0}, C_{0}$ be as in (LR) and $C \subset C_{0} . d(x, C)$ being $x$-continuous, there is $D \in \operatorname{Rci}(t)$ such that $|d(t, C)-d(s, C)|<\varepsilon$ for $s \in D$. Thus, $\sup \{d(s, C) ; s \in D\} \leq d(t, C)+\varepsilon$. Since (LR) holds, we have $d(x, C) \leq d(x, D)+d(t, C)+2 \varepsilon \leq z(x, t)+z(t, y)+2 \varepsilon$, so $z(x, y) \leq z(x, t)+z(t, y)+2 \varepsilon$. Since $\varepsilon$ is arbitrary, we have $z(x, y) \leq z(x, t)+z(t, y)$.

$(2) \Rightarrow(1)$. For some $D_{0} \in \operatorname{Rci}(t)$ and some $C_{0} \in \operatorname{Rci}(y), z(x, t)<d(x, D)+\varepsilon / 3$ for $D \in \operatorname{Rci}(t)$ with $D \subset D_{0}$, and $z(t, y)<d(t, C)+\varepsilon / 3$ for $C \in \operatorname{Rci}(y)$ with $C \subset C_{0}$. Since $d(x, C)$ is $x$-continuous, there is $D_{C} \in \operatorname{Rci}(t)$ with $D_{C} \subset D_{0}$ such that $d(t, C)<d(s, C)+\varepsilon / 3$ for $s \in D \in \operatorname{Rci}(t)$ with $D \subset D_{C}$, and hence, $d(t, C)<\sup \{d(s, C) ; s \in D\}+\varepsilon / 3$. Thus $d(x, C) \leq z(x, y) \leq z(z, t)+z(t, y) \leq$ $d(x, D)+\varepsilon / 3+d(t, C)+\varepsilon / 3<d(x, D)+\sup \{d(s, C) ; s \in D\}+\varepsilon$.

$(2) \Rightarrow(3)$. For a given $\varepsilon>0$, we take $C \in \operatorname{Rci}(y)$ such that $z(x, y)-\varepsilon<z\left(x, y^{\prime}\right)$ by $1.2(4)$, and $z\left(y, y^{\prime}\right)<\varepsilon$ for $y^{\prime} \in C$ by $1.2(3)$. On the other hand, $z\left(x, y^{\prime}\right) \leq$ $z(x, y)+z\left(y, y^{\prime}\right)<z(x, y)+\varepsilon$, so $\left|z(x, y)-z\left(x, y^{\prime}\right)\right|<\varepsilon$.

\section{Bicontinuity and continuity of $z(x, y)$.}

4.1. THEOREM. For $(X, d), d(x, C)$ is $s(x)$-continuous iff $z(x, y)$ is $x$-continuous.

ProOF. $(\Rightarrow)$ Let $\left\{C_{n} \in \operatorname{Rci}(y) ; n \in \mathbf{N}\right\}$ be a base at $y$ with $C_{n+1} \subset C_{n}$. Since $z(x, y)=\lim d\left(x, C_{n}\right),\left|d(x, D)-d\left(x^{\prime}, D\right)\right|<\varepsilon$ implies $\left|z\left(x^{\prime}, y\right)-z(x, y)\right| \leq \varepsilon$ by the condition $(s(x))$ in 1.1 , and hence $z(x, y)$ is $x$-continuous.

$(\Rightarrow)$ Let $a=z(x, y)$. Since $z(x, y)$ is $x$-continuous, for $\varepsilon>0$, there is $U_{1} \in \operatorname{Rci}(x)$ such that $a-\varepsilon<z\left(x^{\prime}, y\right)<a+\varepsilon$ for $x^{\prime} \in U_{1}$. On the other hand, $z(x, y)=$ $\sup \{d(x, C) ; C \in \operatorname{Rci}(y)\}$ implies that $a-\varepsilon<d(x, C) \leq a$ for some $C \in \operatorname{Rci}(y)$. Since $d(x, C)$ is $x$-continuous, $d(x, C)-\varepsilon<d\left(x^{\prime}, C\right)<d(x, C)+\varepsilon$ for $x^{\prime}$ in some $U \in \operatorname{Rci}(x)$ with $U \subset U_{1}$. Thus we have $a-2 \varepsilon<d\left(x^{\prime}, C\right)<a+\varepsilon$, and $d\left(x^{\prime}, C\right) \leq$ $d\left(x^{\prime}, D\right) \leq z\left(x^{\prime}, y\right)<a+\varepsilon$ for $x^{\prime} \in U$ and $C, D \in \operatorname{Rci}(y)$ with $D \subset C$. Since $a-\varepsilon<d(x, C) \leq d(x, D) \leq a$ for $D \subset C$, and $a-2 \varepsilon<d\left(x^{\prime}, D\right)<a+\varepsilon$, we have $\left|d(x, D)-d\left(x^{\prime}, D\right)\right|<2 \varepsilon$, so $d(x, C)$ is $s(x)$-continuous.

4.2. THEOREM. For $(X, d)$, we have $(1) \Rightarrow(2) \Leftrightarrow(3)$.

(1) $d(x, C)$ is locally regular and $s(x)$-continuous.

(2) $z(x, y)$ is bicontinuous.

(3) $d(x, C)$ is $s(x, y)$-continuous.

PROOF. $(1) \Rightarrow(2) . \quad d(x, C)$ being locally regular, by $3.2, z\left(x^{\prime}, y^{\prime}\right) \leq z\left(x^{\prime}, y\right)+$ $z\left(y, y^{\prime}\right)$, and $z(x, y)$ is $x$-continuous. Hence, for $\varepsilon>0$, there is an open set $U \ni x$ such that $\left|z(x, y)-z\left(x^{\prime}, y\right)\right|<\varepsilon / 2$ for $x^{\prime} \in U$. Thus $z\left(x^{\prime}, y^{\prime}\right)<z(x, y)+\varepsilon$, so $\left|z\left(x^{\prime}, y^{\prime}\right)-z(x, y)\right|<\varepsilon$ by $1.2(4)$. Hence $z(x, y)$ is bicontinuous.

$(2) \Rightarrow(3)$. Let $\varepsilon>0$ and $a=z(x, y)$. Since $z(x, y)$ is bicontinuous, there are $U_{1} \in \operatorname{Rci}(x)$ and $C_{1} \in \operatorname{Rci}(y)$ such that $\left|a-z\left(x^{\prime}, y^{\prime}\right)\right|<\varepsilon / 2$ for $x^{\prime} \in U_{1}$ and $y^{\prime} \in C_{1}$. By the definition of $z(x, y), a-\varepsilon / 2<d(x, C) \leq a$ for some $C \in \operatorname{Rci}(y)$ with $C \subset C_{1}$. Since $d(x, C)$ is $x$-continuous, $\left|d\left(x^{\prime}, C\right)-d(x, C)\right|<\varepsilon / 2$ for $x^{\prime} \in U$ for some $U \in \operatorname{Rci}(x)$ with $U \subset U_{1}$. Let $D \in \operatorname{Rci}(y)$ with $D \subset C$ and $E \in \operatorname{Rc}(X)$ with $E \subset C$. Take $y^{\prime} \in$ int $E$. Then we have $d(x, C) \leq d(x, D) \leq a$ and $d\left(x^{\prime}, C\right) \leq$ $d\left(x^{\prime}, E\right) \leq z\left(x^{\prime}, y^{\prime}\right)$. If $d(x, D) \geq d\left(x^{\prime}, E\right), d(x, D)-d\left(x^{\prime}, E\right) \leq a-d\left(x^{\prime}, C\right)<\varepsilon$. If $d\left(x^{\prime}, E\right)>d(x, D), d\left(x^{\prime}, E\right)-d(x, D) \leq z\left(x^{\prime}, y^{\prime}\right)-d(x, C)<\varepsilon$. Thus we have $\left|d(x, D)-d\left(x^{\prime}, E\right)\right|<\varepsilon$.

$(3) \Rightarrow(2)$. For $y^{\prime} \in$ int $C$, apply $E \in \operatorname{Rci}\left(y^{\prime}\right)$ with $E \subset C$ to $E$ in $(s(x, y))$ of 1.1. 


\section{Proof of the main theorem.}

PROOF OF (1). Let $F$ be a closed subset of $X$. Since $d(x, C)$ is $x$-continuous and $z(x, F)=\sup \{d(x, C) ; C \in \operatorname{Rci}(X, F)\}, z(x, F)$ is lower semi-continuous. On the other hand, since $z(x, y)$ is $x$-continuous by 4.1 and $z(x, F)=\inf \{z(z, y) ; y \in F\}$, $z(x, F)$ is upper semi-continuous. Thus $z(x, F)$ is $x$-continuous. Moreover, it is easily verified that $z(x, F)=0 \Leftrightarrow x \in F$ for every closed set $F$ : i.e., $z(x, F)$ is an annihilator of $F$, and that if $E$ and $F$ are closed, and $E \subset F$, then $z(x, E) \geq z(x, F)$ for every $x$; i.e., $z(x, F)$ is monotone. Thus $z(x, F)$ is a continuous monotone annihilator of the family of all closed sets in the sense of Dranišnikov [2], and hence $X$ is stratifiable $[\mathbf{1 , 2}]$, so $X$ is metrizable [3].

PROOF OF (2). By 4.2, $z(x, y)$ is bicontinuous. The second part of (2) follows then directly.

\section{REFERENCES}

1. C. J. Borges, On stratifiable spaces, Pacific J. Math. 17 (1966), 1-16.

2. A. N. Dranisnikov, Simultaneous annihilator of families of closed sets, $\kappa$-metrizable and stratifiable spaces, Soviet Math. Dokl. 19 (1978), 1466-1469.

3. T. Isiwata, Metrization of additive $\kappa$-metric spaces, Proc. Amer. Math. Soc. 100 (1987), 164168.

4. E. V. Scepin, Topology of limit spaces on uncountable inverse spectra, Russian Math. Surveys 31 (1976), 155-191.

5. __, On $\kappa$-metrizable spaces, Math. USSR Izv. 14 (1980), 407-440.

6. J. Suzuki, K. Tamamo and Y. Tanaka, $\kappa$-metrizable spaces, stratifiable spaces and metrizations, Proc. Amer. Math. Soc.

Department of Mathematics, FaCulty of EduCATION, Saitama University, Shimo-OKubo, URAWA, SaItama, JAPAN 